\title{
ALTERNATIVAS DENTRO DE LA EDUCACION FORMAL: el programa Escuela Nueva de Colombia
}

Rosa María Torres*

\section{Introducción}

El programa Escuela Nueva (en adelante EN) de Colombia se ha convertido en referente obligado de las publicaciones educativas de los organismos internacionales; Unesco, Banco Mundial y Unicef, entre los principales organismos, han apoyado decididamente el programa, promoviéndolo y recomendándolo como experiencia modelo. La Unesco ha dicho que "constituye una experiencia de valor internacional innegable", mientras cl Banco Mundial afirma que sus elecciones "merecen diseminarse ampliamente entre los planificadores de la educación y los encargados de la adopción de políticas en el mundo en desarrollo". Misiones de estudio y observación han pasado por Colombia para conocer esta experiencia. Varios países están interesados en la posibilidad de replicarla, dentro y fuera de la región.

¿Qué hace de EN una experiencia tan destacada? En términos generales: 1) el propio hecho de ser EN una innovación y una alternativa dentro del sistema educativo formal, 2) el proceso largo de maduración y desarrollo en el que ésta ha venido configurándose, 3 ) la visión sistémica de la propuesta, 4) el peso puesto sobre el componente curricular y pedagógico, como eje en torno al cual se articula el sistema, y 5) los resultados efectivos que viene mostrando el programa.

Analizaremos, en lo que sigue, estos cinco puntos, tanto en sus fortalezas como en sus debilidades, concluyendo con algunas consideraciones acerca de las posibilidades de replicabilidad de EN, en otros contextos.

\section{Escuela nueva: una alternativa dentro de la educación formal}

Muchos creen -aún en la propia América Latina- que EN es un programa de educación no-formal e, incluso, un programa no-gubernamental. Este es el caso, en efecto, de otros programas destacados por los organismos internacionales como modelos exitosos de educación básica (por ejemplo, el programa de educación primaria rural del BRAC, en Bangladesh). Pero no es el caso de EN. Quizás el mayor mérito y el elemento más inspirador de EN consista, precisamente, en que no se trata de una alternativa a la educación formal ni a la educación estatal sino de una alternativa dentro de los parámetros de la educación formal y la educación pública.

En el campo educativo, lo "alternativo" ha tendido a asociarse con educación no-formal. A ésta se le atribuye una serie de virtudes, por oposición a los defectos que, a su vez, se le atribuyen a la educación formal. Es así como se ha construido la lista conocida de pares opuestos: inflexible/ flexible, vertical/ horizontal, monólogo/ diálogo, irrelevante/ relevante, acrítica/ critica, autoritaria/ participativa, divorciada de la realidad/ vinculada a la realidad, separada de la comunidad/ integrada a la comunidad, etc.

\footnotetext{
* Ecuatoriana. Pedagoga, lingüista y periodista educativa. Investigadora, asesora de diversos programas y autora de numerosas publicaciones sobre temas educativos. Directora de Investigaciones del Instituto Fronesis, de Quito. Actualmente se desempeña como asesora educativa en Unicef-Nueva York.
} 
La educación formal, hoy mundialmente cuestionada por su baja calidad y eficiencia, está, para muchos, desahuciada, al tiempo que cobra auge, se diversifica y expande la educación no-formal. No obstante, ni la una es un dechado de virtudes ni la otra es un manojo de defectos. Dentro de la educación formal vienen dándose cambios importantes. EN de Colombia es, entre otros, un ejemplo de que dicho cambio es posible, de que existen alternativas no sólo a la educación formal sino dentro de ésta.

\section{Escuela Nueva: de proyecto local a política nacional}

Los "proyectos piloto" han perdido credibilidad, dado el alto número de proyectos denominados "piloto" que no han pasado de ser experiencias locales, que se han iniciado y han concluido sin la previsible ampliación o impacto. Al mismo tiempo, proliferan los programas que se implantan de una vez y de manera masiva, o que se expanden aceleradamente, sin haber pasado por un proceso de prueba, maduración y desarrollo. En este contexto, EN resulta una experiencia excepcional. Haber pasado de experiencia micro a política educativa nacional es uno de los elementos destacados y uno de los grandes atractivos del programa.

Los orígenes de EN se remontan a inicios de la década de los 60 , con la implantación de la Escuela Unitaria, promovida, entonces, por la Unesco y adoptada en varios países de América Latina y el Tercer Mundo. La primera Escuela Rural Unitaria en Colombia se montaba en Pamplona, departamento de Santander. El profesor, entonces al frente de la Escuela, llegada a ser el Coordinador Nacional de EN. Para mediados de los 60, la pequeña escuela se había multiplicado en 150 escuelas piloto. En 1967, el gobierno colombiano decidía extender la metodología de la Escuela Unitaria a todas las escuelas unidocentes del país.

En 1975, sobre la base de esta experiencia acumulada, surgía el Programa Escuela Nueva. Entre 1975 y 1978, con apoyo de la Agencia Internacional de Desarrollo (AID), llegaban a funcionar 500 escuelas en tres departamentos. Entre 1982 y 1986 el programa se extendía a la Costa Pacífica. En 1985, con una cobertura nacional de 8.000 escuelas, el gobierno colombiano decidía adoptar a EN como estrategia para universalizar la educación primaria rural. En 1987 comenzó, de este modo, el proceso acelerado de expansión. Para 1989 cubría 17.984 escuelas. En 1991 llegaban a alrededor de 20.000 de las 27.000 escuelas rurales del país, con una cobertura estimada de 1 millón de niños. Un largo proceso de cerca de dos décadas, sumadas, al menos, a otra década de experiencia previa acumulada es, pues el que media entre aquel proyecto local y el actual programa de cobertura nacional y proyección internacional.

\section{Escuela Nueva: una propuesta sistemática}

EN no es -como muchos afirman- una metodología. Es un sistema integrado que combina cuatro aspectos: a) curricular, b) capacitación, e) administrativo, y d) comunitario. Ninguno de estos componentes se explica por si solo. La interrelación entre ellos es lo que da coherencia y factibilidad al modelo.

a) El componente curricular.- Las cuestiones curriculares ocupan un lugar destacado en EN. Elementos claves son la Metodología Activa empleada, los materiales de estudio - llamados Guías de Aprendizaje-, los Rincones de Trabajo, la Biblioteca Escolar, el Gobierno Escolar, y la Promoción Flexible. 
El currículo de EN está orientado a las zonas rurales y a la realidad de la escuela multigrado (uno o dos profesores a cargo de todos los grados de la enseñanza primaria que, en el caso de Colombia, comprende cinco años). Los niños estudian en pequeños grupos usando las Guías, las cuales son entregadas gratuitamente por el Estado. Las Guías están organizadas por áreas (Matemáticas, Ciencias Naturales, Ciencias Sociales y Lenguaje) y por niveles (del segundo al quinto nivel; el primer nivel no cuenta con Guías). Están diseñadas como un material auto-instruccional, con actividades y ejercicios graduados e indicaciones detalladas sobre cómo hacerlos, de modo que los alumnos puedan trabajar, en buena medida, solos, apoyándose entre ellos. De esta manera se busca liberar el tiempo y facilitar la tarea del profesor, reducir las exigencias de calificación docente y permitir que los alumnos avancen a su propio ritmo. A los profesores, en su capacitación, se les enseña a adaptar los contenidos de las Guías a las características especificas de los niños y del medio, así como a las necesidades de la comunidad y a las expectativas de los padres de familia.

Los Rincones de Trabajo se organizan por áreas de estudio y con base en materiales que recogen o elaboran los propios niños, o bien, que son aportados por los padres de familia y la comunidad.

Cada escuela tiene una pequeña biblioteca, la cual está integrada al proceso de aprendizaje y es parte de una estrategia de estímulo a la lectura entre los niños, el maestro y la comunidad. Las bibliotecas están dotadas a la fecha aproximadamente de 70 libros, los cuales incluyen textos de consulta para cada una de las asignaturas, obras de referencia (enciclopedia, diccionario, atlas), literatura, y bibliografía sobre temas vinculados con la comunidad.

EN confiere gran importancia al Gobierno Escolar, instancia de organización escolar de los alumnos y, a partir de la cual se busca involucrar a los niños de manera responsable en la co-gestión de la escuela, iniciarles en la vida cívica y democrática, desarrollar actitudes de cooperación, compañerismo y solidaridad, así como capacidad para liderar, tomar decisiones, hablar en público, y trabajar en grupo. El gobierno escolar - Presidente, Vicepresidente, Secretario, Líderes de Comités y Ayudantes de Nivel- es elegido por los alumnos, replicando los procedimientos de una votación democrática, y es renovado periódicamente de modo que varios niños tengan la oportunidad de pasar por una experiencia de dirección.

La evaluación y la promoción se apartan sustancialmente de los parámetros de la escuela convencional. La evaluación es parte del proceso de aprendizaje, y su función principal es advertir a maestros y alumnos acerca de lo que requiere refuerzo. La promoción a objetivos o grados siguientes es flexible (no automática). Cada niño es promovido al nivel siguiente cuando cumple con los objetivos educativos establecidos, lo que puede tomar más (o menos) de un año escolar regular. Es, pues, la escuela la que se ajusta a los calendarios y necesidades de los niños y de sus familias, y no a la inversa. Si un niño se ausenta temporalmente, puede retomar el estudio sin tener que retirarse de la escuela.

El espacio útil de aprendizaje va más allá del aula. Las escuelas de EN cuentan con una huerta y un jardín. Las canchas deportivas y las instalaciones comunitarias se incorporan como espacios adicionales de la escuela. El espacio interior es aprovechado con una adecuada distribución de los rincones de trabajo, la biblioteca, la cocina, el comedor, las baterías sanitarias. El maestro o maestra cuenta, a menudo, con facilidades 
de vivienda en la propia escuela. El medio natural opera como el principal objeto de estudio, y como el principal proveedor de recursos para la enseñanza y el aprendizaje.

b) La capacitación docente.- En la concepción de EN los maestros pasan a tener una función de facilítadores en relación con sus alumnos - guiando, orientando y evaluando el aprendizaje - y de promotores y organizadores en relación con la comunidad. Ambos roles implican importantes cambios de actitud. Por ello, el cambio actitudinal pedagógico y social- ocupa un lugar central en la formación de los docentes de EN.

La capacitación inicial (maestros nuevos) se hace a través de tres talleres secuenciados - Iniciación, Metodológico, y Organización y Uso de la Biblioteca-, de una semana de duración cada uno. Entre taller y taller hay un intervalo de seis y tres meses, respectivamente, a fin de que en dichos espacios los maestros apliquen en sus escuelas lo aprendido. Haber pasado por el primer taller es requisito para la inclusión de la escuela dentro del programa y para que un maestro pueda empezar a trabajar con él. Un principio básico de EN es replicar en la capacitación de los maestros no sólo los contenidos sino las metodologías y las vivencias que ellos mismos habrán de experimentar en el manejo de su escuela, de la clase y de la relación con sus alumnos.

La capacitación continua se hace a través de los llamados Microcentros Rurales, previstos como instancias de intercambio, actualización y perfeccionamiento permanentes, organizados a iniciativa de los propios maestros, y en los que confluyen grupos de 10 a 15 maestros provenientes de localidades cercanas.

c) El componente administrativo. - - El componente administrativo es el menos abordado en la literatura corriente de y sobre EN. Evidentemente, se trata de un terreno clave y complejo, en el que se juegan factores de orden político e institucional que van más allá de lo meramente administrativo.

Administrar "consiste en orientar más bien que en controlar" (MEN-Unicef, 1990), lo que implica que los agentes administrativos se compenetren, a su vez, con los objetivos y componentes del programa y, en particular, con los aspectos pedagógicos.

EN está concebido como un programa altamente descentralizado. A nivel central, un coordinador y un equipo pequeño (10 personas, en 1991, la mayoría de ellas vinculadas desde los inicios a EN y en posiciones directivas) está encargado de coordinar, diseñar las políticas y las estrategias técnicas, y evaluar la implementación del programa. A nivel departamental, la estructura comprende un Comité Representativo, un Coordinador y un Equipo de Multiplicadores (MEN-Unicef, 1990). A partir de 1987 -que es cuando se puso en marcha el Plan de Universalización de la Educación Primaria Rural y se inició el proceso de expansión de $\mathrm{EN}$ - se introdujo una serie de cambios en la estructura administrativa, enfatizándose la descentralización como estrategia. Se crearon dos estructuras nuevas —un Comité de Universalización a nivel nacional y departamental, y los núcleos educativos - destinados a fomentar la descentralización y el respaldo institucional (MEN-Unicef 1990).

d) La relación escuela-comunidad.- En la concepción de EN, la escuela debe convertirse en centro de información y núcleo integrador de la comunidad. La relación escuela-comunidad debe ser, esencialmente, una relación de mutuo beneficio: los padres de familia y la comunidad integrándose a las actividades escolares, a la vez que la escuela promoviendo acciones orientadas al desarrollo local y al mejoramiento de las condiciones de vida de la población. 
A fin de que el maestro se compenetre mejor con el conocimiento de la comunidad y de la realidad local, EN promueve el manejo de una serie de instrumentos: la Ficha Familiar (datos sobre las familias vinculadas a la escuela), el Calendario Agrícola (información sobre las actividades agrícolas de la zona y su época de realización), el croquis de la localidad y la monografía veredal, todos ellos diseñados con participación de los niños, los padres de familia y la comunidad. Todos estos instrumentos, bajo la responsabilidad del maestro, son importantes insumos para la programación educativa.

De diversas maneras se busca incorporar a los padres de familia a las actividades de aprendizaje de los hijos, a la vez que fomentar en los niños un mayor interés por la vida y el conocimiento de sus padres. La biblioteca, los espacios escolares, las actividades culturales y recreativas, están continuamente abiertos a la participación comunitaria. Los Días de Logros — días en que se presentan los resultados académicos así como los informes de actividades del Gobierno Escolar- se aprovechan para compartir actividades propias de la escuela y actividades de la comunidad.

Las Escuelas Demostrativas, organizadas y distribuidas en cada departamento donde opera el programa, son una verdadera institución dentro de EN. En ellas han de poderse "ver" en funcionamiento estos cuatro componentes según parámetros definidos como ejemplares. Por ello, la visita a una Escuela Demostrativa constituye un elemento clave dentro de la estrategia de motivación y formación docente.

\section{Escuela Nueva: una innovación pedagógica}

Es común que las innovaciones, en el terreno educativo, enfaticen los aspectos organizativos y descuiden los aspectos pedagógicos. Muchas experiencias innovadoras de renombre nacional e internacional son reconocidas como tales a partir de cambios introducidos en la gestión administrativa, en la planificación y evaluación, en la instrumentación de nuevas modalidades de servicio, en la infraestructura, en el uso de los espacios, en la introducción de nuevos contenidos. No obstante, la gestión pedagógica, núcleo definitorio de la calidad de la enseñanza-aprendizaje y enclave en el que converge cualquier posibilidad de transformación educativa queda, a menudo, intocada. EN sobresale, entre otras cosas, por la centralidad que ocupa en el programa la innovación pedagógica.

EN recoge y amalgama diversas vertientes del pensamiento educativo y la práctica pedagógica progresistas, partiendo de la filosofía de la Escuela Unitaria -a su vez inspirada en la Escuela Activa-, algunos de cuyos principios incluían: la defensa de la escuela primaria completa a través de la enseñanza multigrado, instrucción individualizada respetando el ritmo de los alumnos, aprendizaje activo, materiales educativos diseñados para permitir al profesor el manejo de varios grupos simultáneamente, y promoción automática.

La metodología de EN se centra en aprender haciendo, en la articulación entre teoría y práctica, trabajo individual y grupal, juego y estudio, directividad y autonomía. Las actividades didácticas promueven la capacidad de pensar, analizar, investigar y aplicar lo aprendido. Los rincones de trabajo, la biblioteca, el trabajo en pequeños grupos, el uso de materiales auto-instruccionales, el gobierno escolar, los talleres de formación docente, los microcentros rurales: todo ello está pensado en función de un aprendizaje activo, no sólo de los alumnos sino también de los maestros. Las funciones clásicas del maestroinstructor están distribuidas entre las Guías (contenidos y métodos de aprendizaje), la Biblioteca (fuente de consulta complementaria), los Rincones de Trabajo (espacios de 
observación y experimentación), el grupo de alumnos (quienes trabajan colectivamente y se ayudan entre sí) y el Gobierno Escolar (espacio de autoformación en valores y actitudes para la convivencia social y democrática).

La formación docente enfatiza la dimensión pedagógica, así como la motivación y la capacidad para innovar. Los Microcentros reúnen en su concepción, de hecho, una serie de cuestiones consideradas claves en toda estrategia de formación docente: la necesidad de una formación continua y en servicio, basada en el contacto y el trabajo grupal entre docentes, el intercambio de experiencias y el análisis crítico de la propia práctica pedagógica.

El lema de EN "Más y mejor educación primaría para los niños de las zonas rurales" sintetiza este intento por conciliar cantidad con calidad. No se trata, únicamente, de ofrecer a los niños de las zonas rurales mayor acceso sino una mejor educación. El intento de distanciamiento de la cultura pedagógica convencional —vertical, autoritaria, memorística, pasiva- y el logro de mejores resultados de aprendizaje que los de la escuela convencional son una constante y un eje vertebrador en la trayectoria de EN.

\section{Escuela Nueva: un programa con resultados efectivos}

La validez de un programa se juzga, por último, no por su ideario o sus objetivos declarados, sino por sus resultados.

Evaluaciones recientes (Psacharopoulos et.al, 1992) señalan que los alumnos de EN tienen mejores resultados de aprendizaje que los alumnos de las escuelas rurales convencionales (excepto en Matemáticas para el So. grado), así como logros significativos en autoestima, creatividad y actitudes cívicas tales como sentido de cooperación, responsabilidad y solidaridad. EN ha elevado la participación comunitaria en asuntos relacionados con las actividades escolares y ha reducido la probabilidad de deserción de los niños al terminar el quinto grado (no así en el tercer grado). Otro estudio (Rojas y Castillo, 1988) concluye que EN ha logrado incidir significativamente sobre la educación de adultos, la extensión agrícola, las competencias deportivas, las campañas sanitarias y las celebraciones comunitarias.

EN ha cambiado el rostro de la educación rural en Colombia. Está mostrando que es posible no sólo extender la escuela al campo y mejorar sustancialmente su calidad, sino diseñar un modelo educativo pensado específicamente para esa realidad, sin renunciar por ello a la calidad y a la eficiencia. No sólo eso: EN estada mostrando que las tradicionales carencias de las zonas rurales pueden pasar a ser vistas y aprovechadas como elementos positivos: la amplitud de los espacios, el contacto con la naturaleza, los recursos que facilita el medio, la relación con la comunidad, el papel central que asumen la escuela y el maestro en la vida comunitaria, el ambiente de cooperación al que se presta la escuela multigrado, los particulares ritmos de la vida rural, etc.

\section{Debilidades de la Escuela Nueva}

Igual que con otras experiencias innovadoras, destacadas a nivel internacional, existe una tendencia a presentar a EN como una experiencia exenta de problemas. Estos, en cualquier caso, aparecen minimizados. Reconocer contradicciones y debilidades parece verse como reñido con el "éxito". En verdad, la idealización no les hace ningún favor a estos programas - quitándoles, por el contrario, credibilidad- ni a quienes pretenden encontrar en ellos la inspiración y el ejemplo a replican Entre el modelo y la realidad, 
como se sabe, existen siempre grandes distancias. Es mérito de EN y de su equipo coordinador precisamente la apertura, la búsqueda y el esfuerzo constante por identificar y salvar esas distancias.

Una visita de estudio realizada en 1991 a EN en su propio terreno me reveló, en lo personal, la magnitud, tanto de las fortalezas como de las debilidades (ver Torres, 1991). Me he referido hasta aquí a las primeras; me referiré ahora a las segundas.

Todos los componentes y elementos descritos requieren mejoras. El propio equipo central del programa se siente insatisfecho con las guías, la capacitación docente, el gobierno escolar y la relación escuela-comunidad. Las Guías requieren una revisión a fondo (se han hecho tres revisiones hasta la fecha) tanto a nivel de contenidos como de didáctica, particularmente las de Matemáticas y Lenguaje. Muchos contenidos y actividades no parecen adaptados a la realidad y necesidades de un niño rural. Por otro lado, el mecanismo de adaptación introducido en las Guías, si bien muy sugerente e interesante, no está siendo aprovechado por buena parte de los maestros. Hay aún un trecho que recorrer en cuanto al diseño instruccional, todavía demasiado formal y rígido para las necesidades de un material autoformativo como éste.

La capacitación docente tiene déficit, tanto de cobertura como de calidad. Los microcentros Rurales son una estrategia aún no cabalmente comprendida ni establecida en todas partes. El Gobierno Escolar no siempre se organiza ni siempre de la manera prevista. El papel excesivamente directivo o paternalista del maestro, así como la formalidad y los rituales - tan caros a la cultura escolar - terminan muchas veces vaciando de contenido al organismo y al proceso. En la medida que la dinamización de la relación escuela-comunidad depende en alto grado del maestro, sus características, formación y voluntad personales definen la calidad de dicha relación que, a menudo, replica los patrones conocidos de la escuela convencional.

La enseñanza de la lecto-escritura — conocimiento fundamentalmente y terreno en el que se define, en buena medida, el futuro escolar de los niños- sigue siendo una de la debilidades mayores de EN en el ámbito curricular y pedagógico. Las Guías, se ha dicho, cubren a partir del segundo nivel, dejando a los maestros en libertad para escoger los métodos y técnicas de alfabetización que cada cual considere más apropiados ${ }^{1}$. Esto, bien sabemos, es una invitación abierta al tradicionalismo pedagógico y a los métodos obsoletos que prevalecen en este campo. Avanzar con una propuesta renovadora en la enseñanza de la lectoescritura, recuperando el conocimiento que se ha acumulado al respecto en los últimos años en la propia región, es uno de los mayores desafíos que tiene por delante EN.

La nueva relación pedagógica que propugna EN es todavía un objetivo por alcanzar antes que una realidad dada y homogénea. Junto a profesores que han internalizado su nuevo rol docente, coexisten profesores perfectamente tradicionales que reproducen la vieja pedagogía bancaria, mostrando en la práctica la posibilidad de la convivencia entre un ideario educativo progresista y una práctica educativa, conservadora y atrasada. En este caso, pueden aceptarse formalmente los principios y estrategias de EN (pedagogía activa, gobierno escolar, promoción flexible, etc.), y mantenerse intocadas las prácticas escolares que son cuestionadas por esos principios y estrategias. En otras palabras: aquí está precisamente la explicación de cómo EN sobrevive, sigue y avanza, pero aquí,

\footnotetext{
${ }^{1}$ Se han hecho diversos intentos en el pasado (Propuestas metodológicas. manuales, etc.), pero, por diversas razones, ninguno de ellos ha llegado a cuajar. 
también, la explicación de cómo puede estancarse, envejecer y burocratizarse, perdiendo su contenido y su fuerza innovadora.

EN pide de los maestros y de las escuelas dos funciones principales: una pedagógica y otra comunitaria, cuyo balance no es fácil de lograr. En todo caso, lo "demostrativo" de las Escuelas Demostrativas parecería estar privilegiando el rol comunitario, a expensas del rol pedagógico. Ciertamente, la Escuela Demostrativa debería mostrar ambas cosas: la posibilidad de una educación eficiente sin renunciar a su anclaje comunitario y social, y la posibilidad de una fuerte relación escuela- comunidad sin sacrificar la calidad del proceso pedagógico. La posibilidad, en fin, de un buen maestro que, sin descuidar su papel principal como docente, esté dispuesto a convertirse en un buen promotor social.

Uno de los aspectos conflictos que se eluden en la bibliografía sobre EN es la cuestión institucional $\mathrm{y}$, en particular, la relación con el Ministerio de Educación, mareada por recelos y conflictos de diverso tipo, y nunca cabalmente resuelta. Entre el boicoteo abierto y la resistencia pasiva, EN ha debido moverse, muchas veces, contracorriente o en los márgenes del sistema, apoyándose estratégicamente en el respaldo de los organismos internacionales, así como de organizaciones privadas de la propia Colombia. La fragilidad y la falta de legitimidad institucional a nivel de las estructuras estatales pasan a constituir, de hecho, un factor debilitante de la capacidad de consolidación y expansión del programa.

Una larga trayectoria - como la que tiene EN- permite desarrollar y avanzar pero, también, anquilosar y envejecer un programa, si no se toman los recaudos para su continuo rejuvenecimiento. El envejecimiento de Escuela Nueva es un tema recurrente entre sus propios actores y crecientemente enfatizado en el contexto de la etapa de crecimiento por la que está pasando el programa.

La expansión ha traído consigo, en efecto, una acentuación de viejos problemas y una serie de nuevos problemas. Según se afirma (MEN-Unicef, 1990), el "precio de ir a escala" ha implicado "sacrificios inevitables en términos de efectividad y eficiencia" y se ha traducido en "la reducción del número de días dedicados a los talleres de capacitación o la imposibilidad, en algunos lugares, de suministrar las Guías de estudio a tiempo para la celebración de los cursos de capacitación. Una consecuencia de estos problemas es, por supuesto, el debilitamiento del aprendizaje experiencial en la capacitación, sumado al desánimo de los maestros y las criticas al programa". La nueva estructura administrativa surgida de las necesidades de expansión ha entrado en conflicto con los equipos técnicos, no siempre consultados, y ha resultado en un crecimiento acelerado de agentes administrativos, con demandas de capacitación que el programa no está en capacidad de satisfacer. En otros casos, la ausencia de tales mecanismos de organización local ha creado situaciones de ineficiencia e incertidumbre respecto a la expansión y el seguimiento del programa.

Agregaríamos a esto la proliferación de "escuelas demostrativas" que está teniendo lugar dentro del proceso de expansión. Si bien la Escuela Demostrativa es considerada una de las estrategias para mantener la calidad, su masificación parecería estar trayendo consigo efectos contrarios, llevando a una pérdida de significado de lo "demostrativo" y, por tanto, del efecto y el sentido mismo de lo ejemplar. Pretender masificar lo "demostrativo" puede ser precisamente una manera de desvirtuar la excelencia y perder calidad. 


\section{¿Es Escuela Nueva un modelo replicable?}

El tema de la replicabilidad está en el orden el día. En el mareo de la estrategia mundial de "Educación para Todos", en la que acceso y calidad van (al menos en teoría) de la mano, la combinación entre innovación y replicabilidad adquiere gran importancia. A las experiencias innovadoras se les pide ya, no sólo capacidad de expansión, sino flexibilidad y capacidad de adaptación a otros contextos. En esta perspectiva, EN es vista internacionalmente como una experiencia altamente atractiva y prometedora. En esta línea, queremos aportar aquí algunas reflexiones.

En primer lugar, es preciso tener clara la especificidad de EN, tal y como la conocemos y como ha sido desarrollada en Colombia: un programa de educación formal/pública/primaria/rural, fundamentalmente dirigida a la problemática de la escuela multigrado. Estas características, que hacen a la especificidad del modelo, no deben olvidarse al momento de pensar en posibles adaptaciones o variantes del mismo. Como tampoco debe olvidarse que EN es un sistema articulado en torno a cuatro componentes - curricular, capacitación, administración y comunitario-, no un conjunto de elementos sueltos. Estas dos precauciones resultan importantes para quienes, urgidos por las comprensiones superficiales y las recetas fáciles, se sienten tentados a "calcar" esta experiencia para cualquier modalidad o nivel educativo, o bien a entresacar de ella algún elemento particular (Guías de Aprendizaje, capacitación docente, gobierno escolar, promoción flexible, etc.) pretendiendo con ello, equivocadamente, replicar el modelo e introducir cambios sustantivos en el sistema.

Hay una serie de elementos que forman parte consustancial de la historia y la configuración particular de EN, algunos de ellos excepcionales y difícilmente presentes o replicables en otros contextos. "Escuela rural", para empezar, es un concepto con referentes concretos muy diversos. La "escuela rural", de la que nos habla EN de Colombia, es una escuela que cuenta con buenas condiciones de infraestructura y equipamiento. Se trata, en general, de construcciones escolares sólidas, con espacios amplios y agradables, con buenas instalaciones, con mobiliario adecuado. Muchas de ellas tienen facilidades de vivienda para el maestro. En ellas pueden encontrarse cocina, comedor, baños, agua corriente, luz eléctrica, televisión. Escuelas rurales, en fin, privilegiadas en comparación con las que caracterizan comúnmente a los países del Tercer Mundo, incluidos los propios países latinoamericanos ${ }^{2}$.

Colombia es un país lingüísticamente homogéneo; el programa EN cuenta de este modo con una ventaja incomparable, al lidiar con un solo idioma, el español. Este solo hecho simplifica enormemente la tarea - diseño curricular y pedagógico, capacitación docente, producción de materiales, enseñanza y aprendizaje- y confiere determinadas características a todos y cada uno de los componentes del programa. Intentar un modelo semejante en situaciones de bilingüismo o multilingüísmo, como las que predominan en las zonas rurales de muchos países del Tercer Mundo, es aún terreno por explorar.

Según un estudio reciente sobre EN realizado por el Banco Mundial (Psacharopoulos et. al, 1992), los maestros de EN tienen, en su mayoría, formación secundaria o universitaria. Por otra parte, en relación con las escuelas convencionales rurales de la

\footnotetext{
${ }^{2}$ Dentro del denominado Plan de Fomento para la Educación en el Área Rural y los Centros Menores de Población, y con recursos financiados por el Banco Mundial, Colombia emprendió a fines de los 70 e inicios de los 80 una mejora física de las escuelas primarias rurales del país, incluyendo reconstrucción de aulas, suministro de agua potable, unidades sanitarias, mesas y asientos para alumnos y maestros (MEN-Unicef, 1990). 
misma Colombia, EN tiene más maestros viviendo en las instalaciones de la propia escuela. Ambos factores -el nivel de formación previa de los docentes y su permanencia en la escuela - están relacionados positivamente con los logros de los alumnos (la formación universitaria resultó vinculada con mejores resultados cognitivos; la permanencia de los maestros en la escuela resultó vinculada, además, con mejores indicadores de creatividad y actitudes cívicas).

EN se ha moldeado en un proceso prolongado y muy particular. Sobre ello insiste de hecho, la literatura del programa: "En Escuela Nueva se garantizan las condiciones técnicas necesarias, ya que el mismo ha sido diseñado y puesto a prueba durante un periodo de 15 años... Además, el gobierno actual ha proporcionado las condiciones políticas necesarias. Por otra parte, se han logrado crear las condiciones financieras, a través de la asignación de recursos gubernamentales, de un préstamo del Banco Mundial y de la cooperación de Unicef otorgada para mantener la calidad del programa de Escuela Nueva a medida que éste se universaliza" (MEN-Unicef, 1990). ¿Qué países reúnen estas condiciones técnicas, políticas y financieras?

En cuanto a las condiciones técnicas, mencionemos sólo un elemento clave y destacado de EN como son las Guías de Aprendizaje. Elaborar buenos libros de texto, según lo reconoce el propio Banco Mundial, requiere competencias técnicas muy específicas y difíciles de encontrar: "Trasladar las especificaciones de un currículo a la elaboración de buenos textos escolares es algo que requiere un conocimiento experto considerable. Un texto escolar debe tener el nivel apropiado de contenido y de dificultad lectora; ser consistente en el enfoque, el método y la exposición; estar adecuadamente secuenciado; motivar a los alumnos; y, finalmente, estar diseñado de manera que pueda ser utilizado por profesores poco calificados $\mathrm{y}$, al mismo tiempo, permitir a los buenos profesores ir más allá del texto. En el mundo entero, pocos individuos poseen la competencia que se requiere para elaborar buenos libros de texto" (Lockheed y Verspoor, 1991). ¿Cuántos programas pueden contar con este recurso humano y técnico calificado?

Además de los recursos estatales canalizados a través del Ministerio de Educación, EN ha venido recibiendo apoyo financiero sostenido de varias agencias internacionales AID, BID, Unicef, Banco Mundial- así como de algunas organizaciones privadas de Colombia. Los costos estimados de EN son entre $5 \%$ y $10 \%$ más altos que los de las escuelas convencionales (Schiefelbein, 1991), siendo la capacitación docente, al menos, tres veces más costosa (Psacharopoulos et. al, 1992). ¿Es esperable un apoyo financiero semejante en otros países? ¿Es dable pensar en procesos prolongados que vayan más allá de los cortos plazos contemplados por los gobiernos y los organismos internacionales para la concesión de préstamos educativos? ¿Puede la propia EN esperar un apoyo sostenido para seguir expandiéndose y perfeccionar al mismo tiempo el sistema?

En un mundo en el que las políticas y los programas vienen junto con las modas internacionales y terminan junto con los gobiernos nacionales, EN brilla como una experiencia innovadora excepcional que ha logrado mantenerse viva a lo largo de casi dos décadas. ¿Cómo pudo EN sobrevivir los cambios de gobierno y de administración, la inestabilidad política y administrativa que caracterizan a toda América Latina? Quizás a falta de un análisis más elaborado, alguien explica el éxito de EN en términos de "una mezcla de promoción, apoyo estratégico, posición académica de los responsables y simple buena suerte" (Schiefelbein, 1991). El rubro "buena suerte" incluye, sin duda, un conjunto grande de impredecibles, inexplicables e irreplicables. 
Diversos estudios en el mundo entero vienen mostrando que una de las características de los programas exitosos y las escuelas efectivas es el papel que en ellos cumplen determinadas personas individuales, con empuje, visión, capacidad de dirección, mística, perseverancia, etc. Parte de la explicación de EN tiene que ver, en efecto, con individuos concretos. El equipo inicial se ha mantenido relativamente estable y unido. Personas ubicadas en lugares claves y en los momentos precisos han sido decisivas en el desarrollo del programa, no sólo a nivel nacional sino también a nivel intermedio y local. Como se señala, "hoy en día, aún y si Escuela Nueva ha sido institucionalizada en todo el país, el apoyo que ésta recibe en algunas provincias depende en buena medida de las preferencias personales de los administradores locales" (Psacharopoulos et. al, 1992:19).

A EN le tomó 10 años entre su constitución oficial como programa (1975) y su adopción como política educativa nacional (1985), momento a partir del cual empezó su expansión. Este proceso ha seguido tres etapas (MEN-Unicef, 1990): a) aprender a ser efectivo (1975-1978), b) aprender a ser eficiente (1979-1986), y c)aprender a ampliarse (1987 a la fecha). Aún con lo largo y programado de este proceso, y con las condiciones técnicas, políticas y financieras que lo han acompañado, todo indica que EN no estaba preparada para enfrentar el acelerado proceso de expansión por el que está atravesando, no al menos sin poner en riesgo su calidad y consolidación. Si esto sucede con un programa tan trabajado y tan cuidado como EN, ¿qué puede esperarse de programas frágiles, recién nacidos, a los que se les pide ampliación y hasta universalización sin haber cubierto las etapas y los requerimientos indispensables para garantizar su misma supervivencia? La presión que hoy en día ponen gobiernos y organismos internacionales por lograr coberturas, por mostrar resultados y convertirse en modelos exitosos, bien pueden estar contribuyendo a minar las bases mismas de toda posibilidad de cambios y avances sustantivos y sólidos en el terreno educativo.

Es mucho lo que otros países y la propia Colombia tienen aún que aprender de EN, tanto de sus posibilidades como de sus debilidades. Es mucho, también, lo que puede hacerse para consolidar y perfeccionar esta experiencia, cuidándola de los riesgos de la moda y del modelo, fortaleciéndola en su papel doméstico-nacional, precisamente para fortalecerla en su papel externo-internacional. Porque EN muestra con claridad un camino, no necesariamente replicable pero, sin duda, altamente alentador e inspirador, de lo que es posible y fundamental hacer.

Subordinada al imperativo del acceso, la transformación radical que requiere hoy la educaaión puede quedar reducida a retoques superficiales, a modificaciones parciales. Si universalizar la educación es la gran tarea de la época, universalizarla sin transformarla equivale a ofrecer más de lo mismo que viene produciendo deserción, repetición, fracaso escolar, desperdicio de recursos, desprofesionalización, desmoralización, ineficiencia. Transformar la educación formal es, sin duda, el gran desafío. Es preciso desformalizar la escuela, volviéndola flexible, relevante, útil, creativa, amena, sensible a la inteligencia y al pensamiento, respetuosa de la diversidad, atenta a las necesidades de los niños, receptiva a las necesidades de los maestros, abierta a la participación de los padres de familia y la comunidad, responsable por su resultados frente a la sociedad.

Esto es, precisamente, lo que EN viene intentando. Y por eso vale la pena apoyarla, conocerla mejor y aprender de ella. 


\section{Bibliografía}

Lockheed, M. yA. Verspoor, Improving education in developing countries, Oxford University Press, A World Bank Publication, Washington, 1991.

Ministerio de Educación Nacional-Unicef, El Programa de Escuela Nueva: más y mejor educación primaria para los niños de las zonas rurales, Bogotá, 1990.

Psacharopoulos, George, Carlos Rojas y Eduardo Vélez, "Achievement Evaluation of Colombia's Escuela Nueva", en Working Papers. Banco Mundial. Washington, abril 1992.

Rojas, Carlos y Zoraida Castillo, Evaluación del programa Escuela Nueva en Colombia, Instituto SER, Bogotá, 1988.

Schiefelbein, Ernesto, In search of the school of the XXI century: Is the Colombian Escuela Nueva the right path finder? Unesco-Unicef, Santiago, 1991.

Torres, Rosa Maria, Escuela Nueva: una innovación desde el Estado, Instituto Fronesis, Quito, 1991. 